\title{
Intrathecal baclofen trials: complications and positive yield in a pediatric cohort
}

\author{
Charlotte Sayer, MBBS, ${ }^{1}$ Daniel E. Lumsden, MRCPCH, PhD,, Sarah Perides, BSc, ${ }^{1}$ \\ Kylee Tustin, BPhty, MSc, ${ }^{1}$ Sanj Bassi, FRCS, ${ }^{2}$ Jean-Pierre Lin, BSc, MBChB, MRCP(UK), PhD, ${ }^{1}$ \\ and Margaret Kaminska, MD'
}

\begin{abstract}
${ }^{1}$ Complex Motor Disorders Service, Evelina London Children's Hospital, Guy's and St. Thomas' NHS Foundation Trust; and 'Paediatric Neurosurgery, Kings' College Hospital, Kings' College Hospital NHS Foundation Trust, London, United Kingdom
\end{abstract}

\begin{abstract}
OBJECTIVE Intrathecal baclofen (ITB) is an effective management option for childhood hypertonia. Given the potential complications of implanted ITB pumps, trials of ITB are usually performed as part of the workup for ITB pumps. Two methods are used for ITB trials, lumbar puncture (LP) and catheter insertion into the intrathecal space. Little has been written to date on the number of positive trials and complications in trials. This study aimed to report the outcomes and complications in ITB trials for childhood hypertonia (dystonia, spastic, or mixed).
\end{abstract}

METHODS A retrospective case notes review was conducted of all patients who underwent ITB trials at the Evelina London Children's Hospital between 2005 and 2012 (inclusive). Positive trials were defined as a reduction in Modified Ashworth Scale by a minimum of 1 point in at least 2 muscle groups and improvement reported by the caregivers in the areas of goals agreed upon between professionals and the families.

RESULTS Our patient group comprised children with dystonia $(n=7)$, mixed spasticity/dystonia $(n=29)$, spasticity $(n$ $=4)$, and pain $(n=1)$. A total of 47 trials were attempted in 41 children. Forty trials were successfully completed, with 39 being positive. Thirty-three were catheter trials, and 14 were LPs. The overall complication rate in the 47 attempted trials was $53 \%: 61 \%$ in catheter trials, and $36 \%$ in LP trials. This difference was not statistically significant. The most common complications were vomiting $(n=9)$ and CSF leak $(n=4)$. The most serious complication was meningitis $(n=1)$ in a catheter trial. No patients experienced a permanent injury.

CONCLUSIONS There is a high risk of minor self-limiting complications with ITB trials, which needs to be factored into the decision process of progression to trials. The rate of positive trials in this study was $98 \%$, of which $21 \%$ did not progress to pump implantation. While the authors would still advocate for ITB trials prior to ITB pump insertion to aid parental decision-making, this figure suggests that with good patient selection, ITB pumps could be placed without a preceding trial.

http://thejns.org/doi/abs/10.3171/2015.5.PEDS14587

KEY WORDS dystonia; baclofen; intrathecal; children; complications; functional neurosurgery

$\mathrm{B}$ ACLOFEN is a medication commonly used in the management of increased tone. It works as an agonist of the inhibitory neurotransmitter $\gamma$-aminobutyric acid. ${ }^{10}$ Unfortunately, its effectiveness when taken orally is limited due to poor crossing of the blood-brain barrier, leading to high doses being required to combat spasticity or dystonia and concomitant side effects.

Intrathecal baclofen (ITB) has been used since the 1980s for treatment of high tone, especially in the lower limbs. ${ }^{2,11}$ Pump delivery systems allow a patient to receive lower doses of baclofen at a constant rate with fewer side effects than the enteral route. ${ }^{5}$ Placement of an ITB pump is an invasive procedure associated with significant complications; $2,9,14,16,18-20,24,27$ therefore, high-quality patient selection is vital. A common stage of a presurgical workup for the ITB pump is the delivery of an ITB test dose. This enables health care practitioners to confirm the degree of patient sensitivity to the medication and may allow patients and caregivers to appreciate the effect ITB could potentially yield for them. A positive trial often results in an ITB pump being offered, with a negative trial stopping the workup at that point.

ABBREVIATIONS CMDS = Complex Motor Disorders Service; GMFCS = Gross Motor Function Classification System; ITB = intrathecal baclofen; $\mathrm{LP}=$ lumbar puncture; MAS $=$ Modified Ashworth Scale.

SUBMITTED October 25, 2014. ACCEPTED May 11, 2015

INCLUDE WHEN CITING Published online October 30, 2015; DOI: 10.3171/2015.5.PEDS14587. 
There is growing literature regarding the efficacy of ITB pumps for high tone and the complications related to the surgery and ongoing care. To date, little published information is available regarding ITB trials, how they are performed, the incidence of positive/negative trials, and the potential complications or risks. ${ }^{17}$

The 2 methods for conducting ITB trials are lumbar puncture (LP) and an intrathecal catheter. LP is typically performed with the patient awake, which has the advantage of enabling the entire trial to be undertaken within a day without necessitating waiting for the patient to recover from general anesthesia. However, LP access can be traumatic for the child and difficult for the clinician, particularly in the presence of spinal deformity or extensor posturing. ITB trials may need to be repeated at higher doses to ensure a positive effect, such that a patient might require multiple LPs. A catheter trial, on the other hand, typically involves insertion of an intrathecal catheter with the patient under general anesthesia and administration of the test dose once the patient has recovered from the anesthesia. The catheter can be kept in place for repeat doses, if necessary, or it can be used for a continuous infusion via an external pump. ${ }^{8}$ Reports of outcomes and complications of ITB trials are currently limited.

The aim of this study was to report the outcome of ITB trials in a consecutive cohort of children and young people undergoing assessment at our center and to report the incidence and nature of complications arising in the cohort.

\section{Methods \\ Study Participants}

All patients undergoing an ITB trial between 2005 and 2012 (inclusive) were identified from the Complex Motor Disorders Service (CMDS) database at the Evelina London Children's Hospital. Data were collected from case notes and entered on a standardized form. Data collected included sex, age at referral to CMDS, age at referral for the ITB trial, motor phenotype (dystonia, spasticity, or mixed), ${ }^{26}$ age at trial, underlying pathology, Gross Motor Function Classification System (GMFCS) level ${ }^{25}$ (in noncerebral palsy cases a "GMFCS equivalent" score was applied as reported previously by our group ${ }^{15}$ ), the mode of the trial, dose of baclofen, method of scoring of the trial, trial result (positive or negative), all adverse events during admission for the trial and, if applicable, age at referral for pump, age at pump implant, and reason for not having a pump.

At the Evelina London Children's Hospital, patients are offered ITB trials after a thorough assessment by a pediatric neurologist and experienced pediatric physiotherapist during an appointment in the CMDS clinic.

Selection criteria for ITB trials were generalized or segmental hypertonia, predominantly in the lower limbs, interfering with comfort, care, or function; insufficient response to addressing exacerbating factors and other treatment options, such as physiotherapy, occupational therapy, medications, and botulinum toxin; and realistic expectations/goals for intervention.

Caution is applied in cases of unrealistic expectations, significant central hypotonia, respiratory concerns related to upper airway or frequent chest infections, identified risk factors for poor compliance, and in very small patients (i.e., weight below $14-15 \mathrm{~kg}$ ). Primary dystonia is preferentially considered for deep brain stimulation. ${ }^{1}$

\section{Trial Method}

Decisions about the trial mode were typically made in the clinic based on clinical assessment and family preference. LP was offered when it was believed to be clinically feasible to perform the procedure with the child awake. Insertion of the catheter under general anesthesia was planned when there was significant spinal deformity or it was anticipated that the child would be uncooperative and difficult to position, when an anesthetic for brain MRI was planned prior to the trial, or when the family was averse to an awake LP. As observed in our practice, sedative medications or general anesthesia can reduce hypertonia for more than 2 hours, particularly in cases of dystonia; therefore, to avoid unclear trial results, we do not administer test ITB doses with the patient under sedation or general anesthesia (also a reported practice ${ }^{1,17}$ ). Patients were referred to the neurosurgical team for catheter insertion with radiographic guidance when severe spinal deformity was anticipated to prohibit successful access or in the presence of comorbidity such as known hydrocephalus.

Both LP and catheter insertions were performed as sterile procedures following careful skin preparation and using standard LP needles and epidural catheters available at our pediatric hospital. Trials were conducted according to the local protocol by a pediatric neurologist and physiotherapist on the pediatric neurology ward, with an experienced nurse allocated to the patient. LP was performed following application of topical anesthesia using a 22-gauge Quincke spinal needle with patients lying on their side. Once CSF flow was observed from the needle, a dose of ITB was administered from a syringe as a slow push over 1-2 minutes.

For the sterile catheter procedure, an epidural catheter (most commonly 18-gauge, or in smaller patients 24-gauge) was inserted into the intrathecal space while the patient was under general anesthesia and secured with sterile dressing on the skin. The catheters were not tunneled. Most of the catheters were inserted by a pediatric neurologist without available radiographic guidance. In some cases, the catheter was inserted by a neurosurgeon with radiographic guidance. Once the child recovered from the anesthesia, usually the following morning, the catheter site was inspected, and the catheter access port was then aseptically accessed for aspiration of $1 \mathrm{ml}$ of CSF to ensure appropriate catheter position and integrity. The baclofen dose was then slowly administered (over 1-2 minutes) followed by a slow flush of $1-2 \mathrm{ml}$ of $0.9 \% \mathrm{NaCl}$, and then the port was secured. Following drug administration, patients were placed supine with the head elevated $20^{\circ}$ to $30^{\circ}$.

The baclofen dose was weight dependent. If the child weighed less than $20 \mathrm{~kg}$, a starting dose of $25 \mu \mathrm{g}$ was used; for children over $20 \mathrm{~kg}$, the starting dose was $50 \mu \mathrm{g}$. If no response was documented, the dose was escalated by $25 \mu \mathrm{g}$ and repeated daily, aiming for a maximum of 3 administrations. A dose of $12.5 \mu \mathrm{g}$ was used in 1 patient 
who weighed $14.5 \mathrm{~kg}$. We have not used continuous infusion via an external pump.

A pediatric neurologist and a physiotherapist assessed the patients prior to administration of the dose and 2, 4, and 6 hours afterward.

Response to the ITB test dose was evaluated using the Modified Ashworth Scale (MAS) score, ${ }^{7}$ passive range of movement, presence and quality of deep tendon reflexes, and subjective comments from children and caregivers (e.g., related to comfort, ease of care, and positioning). The MAS score was used pragmatically for the assessment of our whole cohort. With the majority of patients having dystonia as a contributing feature, evaluation of their hypertonia with MAS presented assessment challenges. Two experienced professionals performed assessments independently, and a positive response was documented when detected by both raters. A positive trial was defined as the reduction in the MAS score by a minimum of 1 point in at least 2 muscle groups and improvement reported by the patients/caregivers in the areas of goals agreed upon between professionals and the families.

All children remained in the hospital for a minimum of 24 hours after the trial. Fluid intake was carefully monitored, with intravenous fluids given if there was reduced fluid intake or vomiting. Patients were encouraged to lie flat in bed for a minimum of 12 hours after LP and following catheter removal, as well as with the catheter in situ. Any adverse event documented as arising during and following the trial was considered a complication of the trial. Furthermore, any adverse experiences reported by the families within a few days following discharge that were believed to be related to the trial were considered complications.

\section{Results}

A total of 41 children (16 female, 25 male) were identified as having had an ITB trial between 2005 and 2012 (inclusive). The mean age in years at trial was 9, with a range of 3.3 to 17.6 years. Table 1 summarizes the clinical features of the patients; Table 2 summarizes the GMFCS scores.

A total of 47 trials were attempted in 41 patients. Of the 47 trials, 40 (85\%) were successfully completed, with 34 patients $(83 \%)$ having completed the trial on the first attempt. Of the 7 trials that failed on first attempt, 1 (2\%) was a failed LP that was subsequently successful with catheter placement, and $3(6 \%)$ were failed catheter placements by a pediatric neurologist that were later completed after catheter placement under radiographic guidance by a neurosurgeon. In 2 patients, the catheter became dislodged before the trial was started. In both patients, the catheter method had been chosen, because the patients had required general anesthesia for brain and spinal MRIs prior to the trial. These were both successfully converted to LP trials. One catheter was removed due to continued excessive CSF leakage, and the trial was not reattempted at parental request.

The intrathecal catheter was used more commonly than LP bolus. A total of 7 patients required catheter insertion by a neurosurgeon, including the 3 patients with previ-
TABLE 1. Clinical phenotype of patients

\begin{tabular}{lc}
\hline \multicolumn{1}{c}{ Phenotype } & No. of Patients \\
\hline Mixed spasticity/dystonia & 29 \\
\hline Dystonia & 7 \\
\hline Spasticity & 4 \\
\hline Pain & 1 \\
\hline
\end{tabular}

ously failed attempts and 4 patients referred directly to the neurosurgeon (3 due to significant lumbar lordosis and 1 due to hydrocephalus with a ventriculoperitoneal shunt). The complication rate and types of complications for both methods are summarized in Tables 3 and 4.

Although $61 \%$ of patients in the catheter trials and $36 \%$ of patients in the LP trials had complications, the difference in complication rate was not statistically significant (Fisher's exact test, $\mathrm{p}=0.4971$ ).

Of the 40 patients who successfully completed the trial, 1 had a negative response and 39 had positive responses $(98 \%)$, with $31(79 \%)$ of these progressing to ITB pump placement. Eight patients did not progress to the ITB pump despite a positive trial. Reasons included respiratory concerns identified during a sleep study after the trial although no respiratory complications were noted at trial $(\mathrm{n}=2)$, medications trial/alternative therapies improved condition $(n=2)$, parental concern following ITB trial complication of meningitis $(n=1)$, body habitus too small to accommodate a pump $(\mathrm{n}=1)$, awaiting pump placement at the time of data collection $(\mathrm{n}=1)$, and unknown $(\mathrm{n}=1)$.

The child for whom the ITB trial was considered negative presented with evidence of leukodystrophy on MRI of the brain. Clinically, he exhibited minimal hypertonicity of the lower limbs with some upper motor neuron signs present, and pain as the predominant symptom for which ITB was tested. Tone improved following ITB bolus but no improvement in pain was noted according to a numerical rating scale for self-report of pain, and a significant underlying weakness was unmasked. This was therefore classified as a negative response.

The majority of trials were concluded with 1 dose of baclofen, with 11 patients receiving a second higher dose. A third dose was not required in our cohort. A dose of 100 $\mu \mathrm{g}$ was used once and resulted in deep sedation.

\section{Discussion}

In a consecutive cohort of 40 children completing an ITB trial at our institution, we found positive responses to the trial in all but 1 patient and a complication rate of $53 \%$ (61\% for catheter trials and 36\% for LP trials). In all

TABLE 2. Gross Motor Function Classification Score of patients

\begin{tabular}{cc}
\hline GMFCS & No. of Patients \\
\hline 3 & 3 \\
\hline 4 & 5 \\
\hline 5 & 33 \\
\hline
\end{tabular}


TABLE 3. Complications by trial method

\begin{tabular}{lll}
\hline \multicolumn{1}{c}{ Variable } & Catheter & LP Bolus \\
\hline No. of patients & 33 & 14 \\
\hline No. of patients w/ complications (\%) & $20(61)$ & $5(36)$ \\
\hline Complications & & \\
\hline Back pain & 3 & 0 \\
\hline Vomiting & 7 & 2 \\
\hline Dehydration & 1 & 0 \\
\hline CSF leak & 4 & 0 \\
\hline Infection & 1 meningitis) & 1 \\
\hline Dizziness & 1 & 0 \\
\hline Drowsiness & 1 & 0 \\
\hline Headache & 3 & 0 \\
\hline Deep sedation & 2 & 1 \\
\hline Worsened dystonia prior to \& after trial & 2 & 0 \\
\hline Hypotension & 0 & 1 \\
\hline Catheter removal prior to use & 1 & $\mathrm{NA}$ \\
\hline Catheter displacement & 2 & $\mathrm{NA}$ \\
\hline Catheter placement failed & 3 & $\mathrm{NA}$ \\
\hline LP failed & $\mathrm{NA}$ & 1 \\
\hline
\end{tabular}

$\mathrm{NA}=$ not applicable.

patients, complications were conservatively managed, and in no patient did permanent injury arise as a consequence of the trial.

In the majority of children in our cohort, the catheter method was used, compared with LP bolus. Expected difficulty in successfully performing LP bolus without anesthesia due to spinal deformity was the most common indication for a catheter.

Albright and Ferson ${ }^{4}$ described the use of ITB infusion via external pump for trials in patients with dystonia; however, they reported that for younger and smaller children a single bolus might be sufficient to determine a response. We have not used continuous infusions but were ready to consider this method in dystonic patients if a response to bolus was negative.

Although the complication rate did not statistically differ between the 2 trial methods, catheter trials caused patients to commonly have more than 1 complication. The profile of complications differed between the 2 trial types. In the LP bolus method, only 1 child had more than 1 complication, and the only complications described were vomiting, infection (not meningitis or LP-site infection), and sedation associated with a small reduction in blood pressure (short lived with no respiratory compromise). For the catheter group, a greater range of complications was noted, including infection (minor in 2 patients, severe in 1 patient [meningitis]), symptoms related to low intracranial pressure from CSF leakage, and side effects from baclofen itself (e.g., dizziness/drowsiness).

The most severe complication, meningitis, occurred following a catheter trial. The patient developed symptoms of meningitis (fever, headache, vomiting, photophobia, and neck stiffness) within 24 hours of catheter removal. The
TABLE 4. Comparison of multiple complications by trial method

\begin{tabular}{ccc}
\hline $\begin{array}{c}\text { No. of Complications } \\
\text { per Trial }\end{array}$ & $\begin{array}{c}\text { Catheter (no. of } \\
\text { trials/total trials) }\end{array}$ & LP \\
\hline 0 & $13 / 33$ & $9 / 14$ \\
\hline 1 & $13 / 33$ & $4 / 14$ \\
\hline 2 & $2 / 33$ & $1 / 14$ \\
\hline 3 & $4 / 33$ & $0 / 14$ \\
\hline 4 & $1 / 33$ & $0 / 14$ \\
\hline
\end{tabular}

tip of the catheter is routinely sent to the microbiology department for testing and in this case was positive for Staphylococcus aureus. The patient was treated with intravenous antibiotics for 14 days according to sensitivity and made a full recovery. Although the trial had been positive, this complication discouraged the patient's parents from pursuing the ITB pump. Deterioration of dystonia prior to and shortly after the trial was recorded in 2 patients. We believe this deterioration was related to discomfort caused by the presence of a spinal catheter. In both cases, reduction in tone and dystonic spasms was observed following administration of the baclofen test dose.

The patient group reported here is complex, with dystonia contributing to the high tone in the vast majority of the patients. Only 4 patients with pure spasticity were included. In the presence of dynamic posturing, mechanical complications such as catheter displacement may be more likely. The small numbers of patients in different groups reported here preclude a more detailed analysis.

Statistical comparison of the complication rate between each group is limited by the small sample size and the disproportion between group sizes. To be powered to detect a difference in proportions of complications in the 2 groups as demonstrated in our sample at the level of statistical significance, a sample size of 75 in each group would have been required. Assuming that the complication rate in the catheter group represents the "true" complication rate, given the sample size in our study, the analysis was powered only to reach statistical significance if the complication rate in the LP group was below $16 \%$ or greater than $95 \%$. We cannot, therefore, conclude that there is no difference in complication rates, although our results raise the possibility of a trend in this direction.

Complications in ITB trials have been previously reported in articles focusing on the efficacy of ITB, including unilateral radicular pain, ${ }^{20}$ symptoms of low intracranial pressure, ${ }^{6,17,23}$ infection at the skin level at the catheter site, and more severe complications such as positive blood cultures, ${ }^{8,28}$ dizziness, ${ }^{17,28}$ and drowsiness. ${ }^{3,17,19,28}$ Deep vein thrombosis and pulmonary embolism have also been published as a case report. ${ }^{2}$

In a retrospective report by Vloeberghs et al..$^{29}$ that focused on the outcomes of ITB pump implantation in spasticity due to cerebral palsy, $10 \%$ of 85 patients undergoing a trial had spinal tap syndrome with no other complications reported. All of these were catheter trials. Hoving et al. ${ }^{17}$ in a prospective study reported 14 of $17(82 \%)$ patients in their cohort with catheter trials had symptoms 
of lowered CSF pressure, with other complications being reported. Three of 17 patients in whom the catheter was tunneled did not have symptoms of low CSF pressure.

Our overall complication rate was 53\%. In our retrospective study, we included all adverse events recorded in case notes, regardless of whether alternative explanations could be suggested as to the cause of the reported adverse feature. For example, for 2 of the potential infections, short-lasting pyrexia alone was reported, which might relate to other aspects of the hospital stay that exposed our patients to potential infections.

The differences in complication rates can be related to the different trial methods, study design, methodology of data collection, and definition of complications (e.g., individually recorded vomiting, headache, and CSF leak in our study versus spinal tap syndrome ${ }^{23}$ ).

An important finding of this study is the high positive response rate. Of the 40 successfully completed trials, all but 1 child demonstrated a positive response. One patient had a negative trial, for whom reduction of pain was the primary goal rather than tone, the latter responding to baclofen. This suggests a highly selective process for progression to trial. However, $21 \%$ of those did not progress to the ITB pump implant for different reasons, and 1 patient's family refused progression to the pump due to the complications experienced during the trial. There is a similar report by Murphy et al. of parents declining pumps due to complications during the trial of ITB therapy in a pediatric cohort with cerebral palsy. ${ }^{22}$

A high incidence of positive ITB trials has been reported, ${ }^{4,21}$ and $100 \%$ positive trial results have been found in smaller studies. ${ }^{5,13,17}$ Some centers in the US and Europe have abandoned ITB trials due to expected positive response and high complication rates. ${ }^{4,13,21}$ Performing ITB trials was related to complications but was found not to have an effect on risk of dissatisfaction with ITB pump implantation, ${ }^{21}$ although more studies are required to confirm this finding. The expense of trials is significant due to required hospital admission and involved procedures.

Our findings add evidence to support progressing directly to ITB pump implantation in carefully selected patients with hypertonia in specialized centers. However, ITB trials might be useful in ambulant patients and those with complex hyperkinetic movement disorders, where benefits of ITB therapy are less well documented. The ITB trial may remain a useful tool to help patients/families make an informed decision, particularly given the high rate of complications from baclofen pumps ${ }^{2,9,14,16,18-20,24,27}$ and the long-term commitment to meticulous follow-up for pump refills. Additionally, the level of response to the test dose guides the initial ITB daily infusion rate, usually calculated as twice the effective trial dose. Documenting the individual response at the trial might be a useful reference tool if loss of effect from ITB therapy is suspected.

It is important to acknowledge the limitations of this study. This is a relatively small sample that is heterogeneous in terms of etiology and the dominant movement disorder phenotype. It is also a relatively severely affected cohort as seen by the GMFCS levels. Data were retrospectively collected and consequently adverse events attributed to the ITB trial may have had alternative explanations.

\section{Conclusions}

We have reported the outcomes of ITB trials in a consecutive cohort of patients undergoing assessment at our center. A relatively high complication rate was observed. Overall, the complication rate did not statistically differ between the 2 methods used in the ITB trial, although children receiving the catheter demonstrated the potential for multiple complications. The most severe complication, meningitis, occurred in a catheter trial. All but 1 child demonstrated a positive response. These results support proceeding directly to ITB pump implants in patients carefully selected by an experienced multidisciplinary team. Trials might be useful to demonstrate to families the potential gains from ITB therapy. Trials continue to play an important role in the selection process for ambulant patients and hyperkinetic as opposed to hypertonic movement disorders.

The final decision of whether or not to perform the trial should be made together by the involved clinical team and the patient/family carefully considering the indications for the trial while being mindful of the common complications encountered.

\section{References}

1. Albright AL: Intrathecal baclofen for childhood hypertonia. Childs Nerv Syst 23:971-979, 2007

2. Albright AL, Barron WB, Fasick MP, Polinko P, Janosky $\mathrm{J}$ : Continuous intrathecal baclofen infusion for spasticity of cerebral origin. JAMA 270:2475-2477, 1993

3. Albright AL, Cervi A, Singletary J: Intrathecal baclofen for spasticity in cerebral palsy. JAMA 265:1418-1422, 1991

4. Albright AL, Ferson SS: Intrathecal baclofen therapy in children. Neurosurg Focus 21(2):e3, 2006

5. Albright AL, Shultz BL: Plasma baclofen levels in children receiving continuous intrathecal baclofen infusion. J Child Neurol 14:408-409, 1999

6. Bleyenheuft C, Filipetti P, Caldas C, Lejeune T: Experience with external pump trial prior to implantation for intrathecal baclofen in ambulatory patients with spastic cerebral palsy. Neurophysiol Clin 37:23-28, 2007

7. Bohannon RW, Smith MB: Interrater reliability of a modified Ashworth scale of muscle spasticity. Phys Ther 67:206-207, 1987

8. Bonouvrié LA, van Schie PE, Becher JG, van Ouwerkerk WJ, Reeuwijk A, Jeroen Vermeulen R: Effects of intrathecal baclofen on daily care in children with secondary generalized dystonia: a pilot study. Eur J Paediatr Neurol 15:539-543, 2011

9. Brochard S, Remy-Neris O, Filipetti P, Bussel B: Intrathecal baclofen infusion for ambulant children with cerebral palsy. Pediatr Neurol 40:265-270, 2009

10. Brogden RN, Speight TM, Avery GS: Baclofen: a preliminary report of its pharmacological properties and therapeutic efficacy in spasticity. Drugs 8:1-14, 1974

11. Butler C, Campbell S: Evidence of the effects of intrathecal baclofen for spastic and dystonic cerebral palsy. Dev Med Child Neurol 42:634-645, 2000

12. Carda S, Cazzaniga M, Taiana C, Pozzi R: Intrathecal baclofen bolus complicated by deep vein thrombosis and pulmonary embolism. A case report. Eur J Phys Rehabil Med 44:87-88, 2008

13. Dan B, Motta F, Vles JS, Vloeberghs M, Becher JG, Eunson $\mathrm{P}$, et al: Consensus on the appropriate use of intrathecal baclofen (ITB) therapy in paediatric spasticity. Eur J Paediatr Neurol 14:19-28, 2010 
14. Flückiger B, Knecht H, Grossmann S, Felleiter P: Devicerelated complications of long-term intrathecal drug therapy via implanted pumps. Spinal Cord 46:639-643, 2008

15. Gimeno H, Gordon A, Tustin K, Lin JP: Functional priorities in daily life for children and young people with dystonic movement disorders and their families. Eur J Paediatr Neurol 17:161-168, 2013

16. Gray N, Morton RE, Brimlow K, Keetley R, Vloeberghs M: Goals and outcomes for non ambulant children receiving continuous infusion of intrathecal baclofen. Eur J Paediatr Neurol 16:443-448, 2012

17. Hoving MA, van Raak EP, Spincemaille GH, Palmans LJ, Sleypen FA, Vles JS: Intrathecal baclofen in children with spastic cerebral palsy: a double-blind, randomized, placebocontrolled, dose-finding study. Dev Med Child Neurol 49:654-659, 2007

18. Hoving MA, van Raak EP, Spincemaille GH, van KranenMastenbroek VH, van Kleef M, Gorter JW, et al: Safety and one-year efficacy of intrathecal baclofen therapy in children with intractable spastic cerebral palsy. Eur J Paediatr Neurol 13:247-256, 2009

19. Kolaski K, Logan LR: A review of the complications of intrathecal baclofen in patients with cerebral palsy. NeuroRehabilitation 22:383-395, 2007

20. Motta F, Antonello CE: Analysis of complications in 430 consecutive pediatric patients treated with intrathecal baclofen therapy: 14-year experience. J Neurosurg Pediatr 13:301-306, 2014

21. Motta F, Buonaguro V, Stignani C: The use of intrathecal baclofen pump implants in children and adolescents: safety and complications in 200 consecutive cases. J Neurosurg 107 (1 Suppl):32-35, 2007

22. Murphy NA, Irwin MC, Hoff C: Intrathecal baclofen therapy in children with cerebral palsy: efficacy and complications. Arch Phys Med Rehabil 83:1721-1725, 2002

23. Natale M, Mirone G, Rotondo M, Moraci A: Intrathecal baclofen therapy for severe spasticity: analysis on a series of 112 consecutive patients and future prospectives. Clin Neurol Neurosurg 114:321-325, 2012

24. Nielsen JF, Hansen HJ, Sunde N, Christensen JJ: Evidence of tolerance to baclofen in treatment of severe spasticity with intrathecal baclofen. Clin Neurol Neurosurg 104:142-145, 2002
25. Palisano R, Rosenbaum P, Walter S, Russell D, Wood E, Galuppi B: Development and reliability of a system to classify gross motor function in children with cerebral palsy. Dev Med Child Neurol 39:214-223, 1997

26. Sanger TD, Delgado MR, Gaebler-Spira D, Hallett M, Mink JW: Classification and definition of disorders causing hypertonia in childhood. Pediatrics 111:e89-e97, 2003

27. Specchio N, Carotenuto A, Trivisano M, Cappelletti S, Vigevano F, Fusco L: Prolonged episode of dystonia and dyskinesia resembling status epilepticus following acute intrathecal baclofen withdrawal. Epilepsy Behav 21:321-323, 2011

28. van Hilten JJ, Hoff JI, Thang MC, van de Meerakker MM, Voormolen JH, Delhaas EM: Clinimetric issues of screening for responsiveness to intrathecal baclofen in dystonia. J Neural Transm 106:931-941, 1999

29. Vloeberghs M, Keetley R, Morton R: Intrathecal baclofen in the management of spasticity due to cerebral palsy. Pediatr Rehabil 8:172-179, 2005

\section{Disclosure}

D. E. Lumsden, S. Perides, K. Tustin, J. P. Lin, and M. Kaminska have received unrestricted educational grants from Medtronic. J. P. Lin has additionally received consultant payments from Medtronic.

\section{Author Contributions}

Conception and design: Sayer, Lumsden. Acquisition of data: Sayer. Analysis and interpretation of data: Sayer, Lumsden. Drafting the article: Sayer. Critically revising the article: Kaminska, Lumsden, Perides, Tustin, Bassi. Reviewed submitted version of manuscript: all authors. Approved the final version of the manuscript on behalf of all authors: Kaminska. Statistical analysis: Sayer, Lumsden, Lin. Study supervision: Kaminska, Lumsden.

\section{Correspondence}

Margaret Kaminska, Complex Motor Disorders Service, Evelina Children's Hospital, Guy's and St. Thomas' NHS Foundation Trust, Westminister Bridge Rd., London SE1 7EH, United Kingdom. email: margaret.kaminska@gstt.nhs.uk. 